\title{
Hybrid model of clot formation in flow
}

\author{
A. Tosenberger ${ }^{1, a}, \mathrm{~N}$. Bessonov ${ }^{2}$, and V. Volpert ${ }^{3,4}$ \\ ${ }^{1}$ Unité de Chronobiologie Théorique, Faculté des Sciences, Université Libre de Bruxelles (ULB), Campus \\ Plaine, CP 231, Brussels B-1050, Belgium \\ ${ }^{2}$ Institute of Mechanical Engineering Problems, 199178 Saint Petersburg, Russia \\ ${ }^{3}$ Institut Camille Jordan, UMR 5208 CNRS, University Lyon 169622 Villeurbanne, France \\ ${ }^{4}$ INRIA Team Dracula, INRIA Antenne Lyon la Doua, 69603 Villeurbanne, France
}

\begin{abstract}
The process of blood coagulation and clot formation in vivo is not yet completely understood. One of the main questions related to haemostasis is why the clot stops growing in normal conditions before it completely obstructs the flow in the vessel, whereas, in pathologic cases, it can continue to grow, often with fatal consequences. Hence, revealing the mechanisms by which the clot grows and stops growing in the flow remains of great importance. In order to study this topic we have developed a hybrid DPD-PDE method where Dissipative Particle Dynamics (DPD) is used to model plasma flow and platelets, while the protein regulatory network is described by a system of partial differential equations. The model describes the interaction between blood flow, platelet aggregation and plasma coagulation. As a result of modelling we propose a new mechanism of clot growth and growth arrest in flow. The developed model and its parts can be used as a base to modelling of different physiological phenomena related to cell-cell interactions and blood flows.
\end{abstract}

\section{Introduction}

We develop a hybrid model of blood coagulation in flow by coupling two different methods of description - discrete and continuous. The discrete sub-model is based on a particle simulation method called Dissipative Particle Dynamics (DPD) and is used to describe blood flow, blood cells and their interactions. The continuous sub-model is a system of advection-reaction-diffusion equations which describes the coagulation regulatory network and concentrations of corresponding blood factors. Such approach offers a possibility to study interactions between blood flow, coagulation regulatory network, and blood cells. In the two following sections we briefly describe each of sub-models (for more details see [6-8]).

\section{Coagulation sub-model}

We consider the process of blood coagulation in vivo which is characterized by two consecutive phases. In the first phase the coagulation process is initiated (due to a physical injury or other pathological condition) by tissue factor $(T F)$ which is expressed in endothelial cells at the inner surface of

\footnotetext{
ae-mail: alen.tosenberger@ulb.ac.be
} 


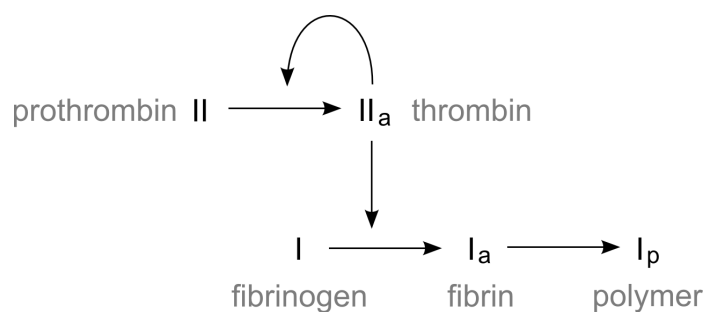

Figure 1. A simplified scheme of the coagulation cascade showing the characteristic reactions.

blood vessels. During the initiation phase the presence of tissue factor leads to the activation of a small but critical amount of prothrombin $(I I)$ to thrombin $\left(I I_{a}\right)$. Although being a very potent coagulant, during this stage thrombin concentration remains low, while the secreted tissue factor is rapidly inhibited by a protein called Tissue Factor Pathway Inhibitor (TFPI). As the effect of tissue factor is limited it is necessary to sustain the production of thrombin in order to create the blood clot. Hence, the initiation phase is followed by the amplification phase in which the activation of prothrombin to thrombin is accelerated through a self-accelerated process. Among many functions, the main purpose of thrombin in coagulation is to act as the enzyme in the activation of fibrinogen $(I)$ to fibrin $\left(I_{a}\right)$, which then polymerizes $\left(I_{p}\right)$ and creates a net-like structure solidifying the blood plasma. The explosive increase of thrombin concentration leads to the rapid generation of fibrin and finally to the creation of a stable clot. The described phases and reactions can be considered as the main characteristics of the coagulation in blood plasma, however, the whole regulatory network is much more complex involving about 30 different blood factors and complex reactions. In our work we consider a simplified scheme (Figure 1) and a qualitative model corresponding to the main characteristics of the regulatory network. Here we show the minimal model including two partial differential equations for concentrations of thrombin and fibrinogen, and one ordinary differential equation for the concentration of fibrin.

$$
\begin{aligned}
& \frac{\partial I I_{a}}{\partial t}+\mathbf{v} \cdot \nabla I I_{a}=D_{I I_{a}} \Delta I I_{a}+k_{1}\left(I I_{a}\right)\left(C_{I I}-I I_{a}\right)-s_{1} I I_{a} \\
& \frac{\partial I}{\partial t}+\mathbf{v} \cdot \nabla I=D_{I} \Delta I-k_{2}\left(I I_{a}\right) I\left(C_{I_{p}}-I\right) \\
& \frac{\partial I_{p}}{\partial t}=k_{2}\left(I I_{a}\right) I\left(C_{I_{p}}-I\right)
\end{aligned}
$$

where $I I_{a}, I$ and $I_{p}$ are concentrations of thrombin, fibrinogen and fibrin polymer respectively, $\mathbf{v}$ is the velocity field obtained from the flow simulations (see the following section), $D_{I I_{a}}$ and $D_{I}$ are diffusion coefficients, $C_{I I}$ is the initial concentration of prothrombin, $s_{1}$ is the thrombin degradation/inhibition rate, and $C_{I_{p}}$ is the maximal concentration of fibrin polymer. The rates of production of thrombin and fibrin polymer are given by the functions $k_{1}$ and $k_{2}$ respectively:

$$
k_{1}\left(I_{a}\right)=k_{1}^{0} \frac{I I_{a}{ }^{2}}{T_{0}+I I_{a}}, \quad k_{2}\left(I I_{a}\right)=k_{2}^{0} I I_{a},
$$

where $k_{1}^{0}, T_{0}$ and $k_{2}^{0}$ are some constants. This minimal model can be extended to take into account other, more detailed aspects of the coagulation cascade related to specific pathologies. 


\section{Blood flow sub-model}

The discrete sub-model describes the flow of blood cells immersed in blood plasma. The sub-model is based on the Dissipative Particle Dynamics (DPD) method, which is a meso-scale particle method suitable for simulating media with hydrodynamic properties. In DPD, three pair-wise forces act between each two particles in some relative proximity defined by a force cut-off radius (for more details see $[3,4,7])$. In our model each DPD particle describes either a single drop of blood plasma or a single platelet. A more detailed model of blood cells is also possible, however, it increases significantly the number of DPD particles and with it the computational cost of simulations. In that case each blood cell is modelled by a deformable membrane model $[1,2,4]$ where the cell membrane consists of DPD particles between which we impose elastic forces.

In the simple model, individual platelets are modelled as single DPD particles and we describe their interactions and aggregation. As each DPD particle (plasma or platelet) describes a certain volume, we can consider a spherical model where the volume is defined by the radius of the sphere. We chose the level of DPD description such that the volume of all DPD particles is the same and their diameter is equal to the radius of platelets $d_{P}$. To describe different types of adhesion between platelets we introduce an adhesion force which acts between connected platelets:

$$
\mathbf{F}_{i j}^{A}= \begin{cases}f^{A}\left(t_{i j}\right)\left(1-\frac{r_{i j}}{d_{P}}\right) \hat{\mathbf{r}}_{i j}, & \text { if } d_{P} \leq r_{i j} \leq d_{D}, \\ \mathbf{0}, & \text { if } r_{i j}<d_{P} \text { or } r_{i j}>d_{D},\end{cases}
$$

where where $\mathbf{r}_{i}$ is the vector of position of the particle $i, \mathbf{r}_{i j}=\mathbf{r}_{i}-\mathbf{r}_{j}, r_{i j}=\left|\mathbf{r}_{i j}\right|, \hat{\mathbf{r}}_{i j}=\mathbf{r}_{i j} / r_{i j}, d_{P}$ is the platelet diameter, $t_{i j}$ is the age of the connection between the platelets, while $d_{D}$ (such that $d_{D}>d_{P}$ ) is the critical distance after which the connection between two platelets is considered to be severed. The function $f^{A}\left(t_{i j}\right)$ describes strengths of different types of inter-platelet bindings:

$$
f^{A}\left(t_{i j}\right)= \begin{cases}k_{1}^{A}, & \text { if } I_{p}(i) \text { or } I_{p}(j)<c_{I_{p}}, \quad \text { and } \quad t_{i j}<t_{c}, \\ k_{2}^{A}, & \text { if } I_{p}(i) \text { or } I_{p}(j)<c_{I_{p}}, \quad \text { and } \quad t_{i j} \geq t_{c}, \\ k_{3}^{A}, & \text { if } I_{p}(i) \text { and } I_{p}(j) \geq c_{I_{p}},\end{cases}
$$

where $f_{1}^{A}<f_{2}^{A}<f_{3}^{A}$ are the three strengths of inter-platelet connections, representing respectively a weak bond due to GPIb receptors, a medium bond due to platelet activation and a strong bond due to reinforcement by fibrin polymer net. The platelet activation process is here approximated by the activation period $t_{c}$ which represents the time needed for platelet activation measured from the moment of the connection establishing. $I_{p}(i)$ and $I_{p}(j)$ are levels of fibrin polymer $I_{p}$ at the positions of platelets $i$ and $j$ respectively. The levels of $I_{p}$ are given by the coagulation sub-model (system of equations (1)). $c_{I_{p}}$ is the critical level of fibrin polymer. A platelet is considered to be a part of the clot core if it is in the clot and the fibrin concentration is higher than $c_{I_{p}}$ at the position of that platelet. Therefore, the part of clot which covered by a concentration of $I_{p}$ larger than $c_{I_{p}}$ can be considered as the stable clot core. As platelets which are coated in fibrin become resistant to further adhesion [5], in the model we impose that platelets of the clot core cannot establish new connections.

The main result of the model is a possible new mechanism of clot growth and growth arrest in flow, presented in Figure 2. Simulation snapshots show the following stages of the clot growth: a) the clot begins to grow by formation of a platelet aggregate, b) some of aggregated platelets activate reinforcing the clot and protecting the concentrations of blood factors from the flow, c) fibrin begins to cover the growing clot allowing the clot to grow further until it reaches the critical size, d,e) parts of clot not covered by the fibrin net rupture and are taken by the flow, f) the last rupture leaves only the adhesion resistant clot core, which prevents the clot from growing further. 

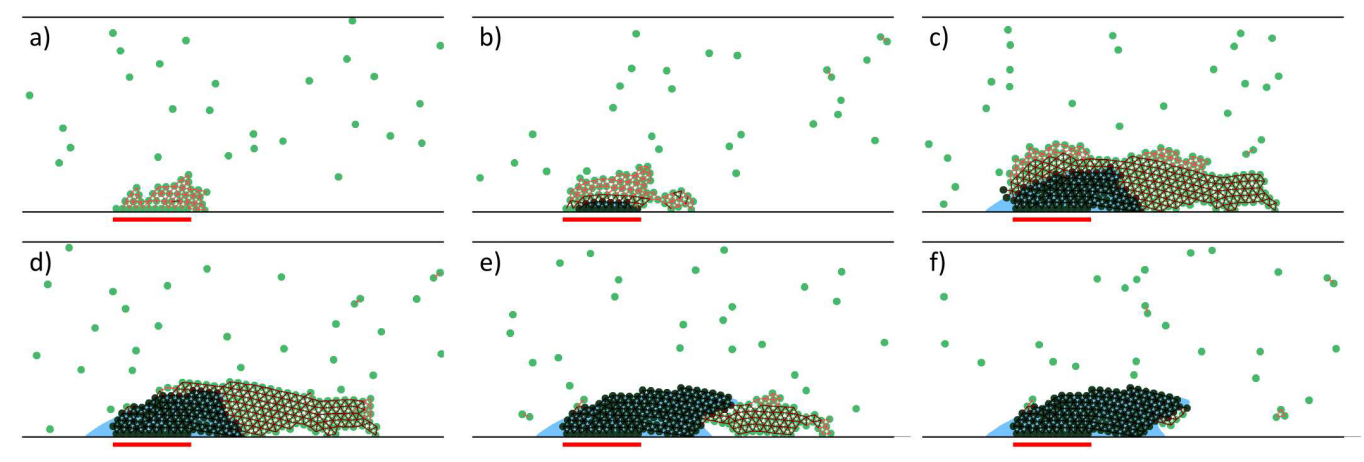

Figure 2. Example of clot growth and growth arrest. Flow is from left to right, platelets are shown in green (dark green if covered by fibrin polymer), fibrin polymer in blue and the platelet connections in red (light red for the weak bonding, dark red for activated platelets, and black for fibrin reinforced bonding). The red line marks the damaged part of the vessel wall.

\section{Discussion}

The model we present here is intended to be the minimal qualitative model which allows studying of interactions between blood coagulation, blood cells and blood flow. Using the same hybrid approach the model can be extended to study in more details the process of blood coagulation, blood flow phenomena [1, 2], and blood related pathologies with local effects [4]. Any increase in details described by the model and especially an increase in the number of DPD particles lead to a significant increase of the simulation cost. The computation cost can be reduced by several optimizations of the algorithm and by asynchronous parallel computing (CPU clusters). However, in this minimal form the model is not suited for synchronous parallel computing (GPGPU) (see [6] p. 122-128).

\section{References}

[1] N. Bessonov, E. Babushkina, S.F. Golovashchenko, A. Tosenberger, F. Ataullakhanov, M. Panteleev, A. Tokarev, V. Volpert, Russ J Numer Anal Math Model 28 (5), 443-458 (2013)

[2] N. Bessonov, E. Babushkina, S.F. Golovashchenko, A. Tosenberger, F. Ataullakhanov, M. Panteleev, A. Tokarev, V. Volpert, Math Model Nat Phenom 9 (6), 69-84 (2014)

[3] D.A. Fedosov, I.V. Pivkin, G.E. Karniadakis, J Comput Phys 227, 2540-2559 (2008)

[4] D.A. Fedosov, Multiscale Modeling of Blood Flow and Soft Matter, PhD dissertation at Brown University, USA (2010).

[5] M.M. Kamocka, J. Mu, X. Liu, N. Chen, A. Zollman, B. Sturonas-Brown, K. Dunn, Z. Xu, D.Z. Chen, M.S. Alber, E.D. Rosen, J Biomed Opt 15 (1): 016020 (2010).

[6] A. Tosenberger, Blood flow modelling and applications to blood coagulation and atherosclerosis, Doctoral dissertation at the University Claude Bernard Lyon 1, Lyon, France (2014)

[7] A. Tosenberger, F. Ataullakhanov, N. Bessonov, M. Panteleev, A. Tokarev, V. Volpert, J Math Biol, DOI: 10.1007/s00285-015-0891-2 (2015)

[8] A. Tosenberger, N. Bessonov, V. Volpert, Math Model Nat Phenom 10 (1), 36-47 (2015) 